\title{
Relationship of elevated casual blood glucose level with coronary heart disease, cardiovascular disease and all-cause mortality in a representative sample of the Japanese population. NIPPON DATA80
}

\author{
S. Kadowaki • T. Okamura • A. Hozawa • T. Kadowaki • \\ A. Kadota • Y. Murakami • K. Nakamura • S. Saitoh • \\ Y. Nakamura $\cdot$ T. Hayakawa $\cdot$ Y. Kita $\cdot$ A. Okayama \\ H. Ueshima $\cdot$ for the NIPPON DATA Research Group
}

Received: 10 July 2007 / Accepted: 22 November 2007 / Published online: 16 January 2008

(C) Springer-Verlag 2007

\begin{abstract}
Aims/hypothesis High fasting blood glucose is one of the well-known risk factors for CHD. However, in certain settings, patients cannot always be expected to fast. For example, community screenings for cardiovascular disease (CVD) risk factors in Japan are performed under non-fasting conditions to achieve high participation rates. Thus, we examined a representative cohort of the Japanese population ( $n=9,444$, follow-up period 17.3 years) to clarify whether high casual blood glucose (CBG) can predict CVD mortality. Methods We defined CBG groups as follows: high $\mathrm{CBG} \geq$ $11.1 \mathrm{mmol} / \mathrm{l}$ or participants with a history of diabetes
\end{abstract}

Electronic supplementary material The online version of this article (doi:10.1007/s00125-007-0915-6) contains a full list of the NIPPON DATA research group members, which is available to authorised users.

S. Kadowaki $(\bowtie) \cdot$ A. Hozawa $\cdot$ T. Kadowaki $\cdot$ A. Kadota $\cdot$

Y. Murakami $\cdot$ K. Nakamura $\cdot$ Y. Kita $\cdot$ H. Ueshima

Department of Health Science,

Shiga University of Medical Science,

Seta Tsukinowa-cho,

Otsu 520-2192, Japan

e-mail: sayakado@belle.shiga-med.ac.jp

T. Okamura

Department of Preventive Cardiology,

National Cardiovascular Center,

Suita, Japan

\section{A. Kadota}

Department of Internal Medicine,

Shiga University of Medical Science,

Otsu, Japan mellitus; borderline high, $7.77 \leq \mathrm{CBG}<11.1 \mathrm{mmol} / \mathrm{l}$; higher normal, $5.22 \leq \mathrm{CBG}<7.77 \mathrm{mmol} / 1)$; and lower normal, $\mathrm{CBG}<5.22 \mathrm{mmol} / \mathrm{l}$. The multivariate-adjusted hazard ratios (HRs) for CHD, CVD and all-cause mortality were calculated.

Results The crude CHD mortality rate was 0.84 per 1,000 person-years. Age- and sex-adjusted HRs for CHD mortality were high among participants with CBG levels $\geq 7.77 \mathrm{mmol} / 1$, regardless of time since last meal. Multivariate-adjusted HRs $(95 \% \mathrm{CI})$ of CHD mortality in high and borderline high CBG groups were $2.62(1.46-4.67)$ and 2.43 (1.29-4.58), respectively. Similar results were observed for both CVD

S. Saitoh

Second Department of Internal Medicine,

Sapporo Medical University,

Sapporo, Japan

Y. Nakamura

Cardiovascular Epidemiology,

Kyoto Women's University,

Kyoto, Japan

T. Hayakawa

Department of Hygiene and Preventive Medicine,

School of Medicine, Fukushima Medical University,

Fukushima, Japan

A. Okayama

The First Institute for Health Promotion and Health Care, Japan Anti-Tuberculosis Association,

Tokyo, Japan 
and all-cause mortality. Even within the normal blood glucose range, each $1 \mathrm{mmol} / 1$ increase in CBG was associated with a statistically significant increase in the HR for CVD mortality $(1.12,95 \%$ CI 1.02-1.22). Population-attributable fractions of the combined groups of high and borderline high CBG for CHD, CVD and all-cause mortality were 12.0, 4.9 and 3.5\%, respectively.

Conclusions/interpretation Increases in $\mathrm{CBG}$, even within the normal range, predict CVD mortality.

Keywords Cardiovascular disease - Casual blood glucose . Cohort study · Coronary heart disease · Diabetes mellitus . Japanese $\cdot$ Mortality $\cdot$ Population-attributable fraction

$\begin{array}{ll}\text { Abbreviations } & \\ \text { CBG } & \text { casual blood glucose } \\ \text { CVD } & \begin{array}{l}\text { cardiovascular disease } \\ \text { hazard ratio }\end{array} \\ \text { HR } & \begin{array}{l}\text { International Classification } \\ \text { of Diseases } \\ \text { ICD }\end{array} \\ \text { NIPPON DATA80 Inal Integrated Project } \\ \text { for Prospective Observation } \\ \text { of Non-communicable Diseases } \\ \text { and its Trends in the Aged, 1980 } \\ \text { population-attributable fraction }\end{array}$

\section{Introduction}

Impaired glucose tolerance and diabetes mellitus are wellknown risk factors for coronary heart disease (CHD) and cardiovascular disease (CVD) [1-4]. A fasting blood glucose level or a fasting blood glucose level plus $2 \mathrm{~h}$ post-glucose load (75 g OGTT) are usually used to diagnose diabetes mellitus [5]. However, in some settings, it is unrealistic to require all participants to fast. It is impractical to require either of these tests from clinic patients, especially those who visit at night or in the afternoon. Furthermore, in Japan, general screenings for CVD risk factors are performed under non-fasting conditions to improve the participation rates. Thus, the question of whether a casual blood glucose (CBG) level, whose value can be obtained at any time of the day regardless of time since last meal, can predict CHD or CVD mortality is of great interest. Only one prospective study has reported this relationship between CBG and CHD [6], though some prospective studies have reported the relationship between CHD and fasting blood glucose or OGTT [7-9]. We investigated the relationship of CBG with CHD, CVD and all-cause mortality in a 17.3-year follow-up study with a representative sample from the Japanese population. We also investigated whether a CBG level predicts CHD or CVD mortality within a normal glucose range and examined what proportion of CHD or CVD deaths was attributable to high $\mathrm{CBG}$ or borderline high $\mathrm{CBG}$ levels.

\section{Methods}

Population The cohort studies of the National Survey on Circulatory Disorders 1980 were called the National Integrated Project for Prospective Observation of Noncommunicable Diseases and its Trends in the Aged, 1980 (NIPPON DATA80). Details of these cohorts have been previously reported [10-14]. Briefly, 13,771 participants were randomly selected in 300 districts from the overall population aged 30 years or older in Japan by the Japanese Ministry of Health and Welfare. Among them, 10,546 individuals completed a baseline survey, namely, the National Survey of Circulatory Disorders, in 1980. All participants were assured a right to refuse the participation in this survey. The participation rate in this survey was $76.6 \%(10,546$ of 13,771). They were followed-up until 15 November 1999 by using the national Vital Statistics. Of the 10,546 participants, 1,102 were excluded for the following reasons: past history of CHD $(n=45)$ or stroke $(n=108)$; information missing at the baseline survey $(n=$ 41 ); or lost to further contact due to incomplete residential access information at the first survey $(n=908)$. The remaining 9,444 participants (4,134 men, 5,310 women) were included in the analysis.

Baseline examination Blood was drawn from seated patients into a plain, siliconised glass tube and the serum was separated. The serum was centrifuged soon after blood coagulation at $1,500 \times \mathrm{g}$. Fasting was not required prior to blood draw. The blood was gathered and analysed at one specific laboratory (formerly Center for Adult Diseases, Osaka; now named Osaka Medical Center for Health Science and Promotion, Osaka, Japan). Since April 1975, this laboratory has been certified by the CDC-NHLBI Lipid Standardization Program of the Center for Disease Control and Prevention (Atlanta, GA, USA) [15]. Blood glucose levels were measured at this site using the cupric-neocuproine method [16] between 1975 and 1986 [17]. Because blood glucose levels are now widely measured with the hexokinase method, the serum glucose levels were adjusted by using a formula $([0.047 \times($ glucose concentration in $\mathrm{mg} / \mathrm{dl})]-0.541)$ previously reported by the same laboratory, which gives levels in $\mathrm{mmol} / \mathrm{l}$ [17]. Iso et al. [17] reported that this formula was obtained from 60 random samples of blood with the regression line $\left(r^{2}=0.93\right)$. Total cholesterol levels were also measured. BMI was calculated as weight $(\mathrm{kg})$ divided by the square of height $(\mathrm{m})$. Obesity was defined as $\mathrm{BMI} \geq 25 \mathrm{~kg} / \mathrm{m}^{2}$ [18]. Public health nurses obtained information about past history of diabetes mellitus, time since last 
meal, smoking, drinking and medication histories. The nurses measured baseline blood pressure using a standard mercury sphygmomanometer on the right arm of seated participants after a 5 min rest. Hypertension was defined as systolic blood pressure $\geq 140 \mathrm{mmHg}$, diastolic blood pressure $\geq 90 \mathrm{mmHg}$, the use of antihypertensive agents or any combination of these. Residential districts were classified as urban, suburban, sub-rural and rural based on the population size of the municipality in which the participants lived [19].

Follow-up survey For deceased participants, the underlying cause of death obtained from National Vital Statistics was coded according to the International Classification of Diseases (ICD), using the 9th revision (ICD9) for the period between 1980 and 1994, and the 10th revision (ICD10) for the period between 1995 and 1999. Deaths from CHD (ICD9: 410-414, ICD10: I20-I25), all heart diseases (ICD9: 410-429, ICD10: I20-I25, I30-I52) and CVD (ICD9: 390-459, ICD10: I00-I99) were defined according to ICD9 and ICD10 codes. The details of the classification in the present study have been described elsewhere [20]. Permission to use National Vital Statistics was obtained from the Management and Coordination Agency, Government of Japan. Approval for this study was obtained from the Institutional Review Board of Shiga University of Medical Science (number 12-18, 2000).

Statistical analysis Participants were divided into the following three groups according to their CBG levels: (1) less than $7.77 \mathrm{mmol} / 1$ (normal); (2) 7.77 to $<11.1 \mathrm{mmol} / 1$ (borderline high $\mathrm{CBG}$ ); and (3) $\geq 11.1 \mathrm{mmol} / 1$ or patients with a history of diabetes mellitus (high $\mathrm{CBG}$ ). In some analyses, the 'normal' $\mathrm{CBG}$ group was divided into two groups at their median, i.e. higher normal (CBG 5.22 to $<7.77 \mathrm{mmol} / \mathrm{l}$ ) and lower normal $(\mathrm{CBG}<5.22 \mathrm{mmol} / \mathrm{l})$. The 'borderline high CBG' and 'high $\mathrm{CBG}$ ' groups were categorised on the basis of OGTT criteria [21]. According to the OGTT criteria, blood glucose levels from 7.77 to $<11.1 \mathrm{mmol} / 1$ and $\geq 11.1 \mathrm{mmol} /$ 1 at $2 \mathrm{~h}$ after glucose intake are defined as impaired glucose tolerance and diabetes mellitus, respectively. In some analyses, we combined higher normal and lower normal into one category.

The risk characteristics of each group at the baseline survey and cause-specific mortality were described as means, standard deviation (SD) for continuous variables and proportions for categorical variables. Analysis of variance was used for comparisons of multiple group means and the $\chi^{2}$ test was used to compare proportions.

We calculated age- and sex-adjusted hazard ratios (HRs) for CHD deaths for the participants whose CBG levels were $\geq 7.77 \mathrm{mmol} / \mathrm{l}$ by comparing the death rate with that for the group whose CBG levels were $<7.77 \mathrm{mmol} / \mathrm{l}$. We divided the participants into four groups by time since last meal to examine the effect of that time on CHD deaths. The four groups were defined as $1,2,3-4$, and $\geq 5 \mathrm{~h}$ since the last meal. The multivariate-adjusted HRs of all four blood glucose categories for CHD, CVD, all-heart or all-cause morality were calculated using a Cox proportional hazard model adjusted for age, total cholesterol, BMI, hypertension, smoking categories (never smokers, past smokers, smoking $\leq 20$ cigarettes per day or smoking $\geq 21$ cigarettes per day), drinking categories (never drinkers, past drinkers, occasional drinkers or everyday drinkers) and residential districts. We applied dummy variables to the smoking and drinking categories. We defined the lower normal group (i.e. $\mathrm{CBG} \leq 5.22 \mathrm{mmol} / \mathrm{l}$ ) as the reference group. We included sex in the model when analysing the combined dataset of men and women. The trend tests were performed by allocating scores 1, 2, 3 and 4 for all participants in lower normal, higher normal, borderline high CBG and high CBG, respectively. To assess whether the positive relationship between CBG and CHD or CVD mortality was observed in participants within the normal blood glucose range, we analysed the relationship between CBG (continuous levels) and CHD or CVD mortality in participants whose blood glucose levels were $<7.77 \mathrm{mmol} / \mathrm{l}$. Additionally, we divided the participants whose blood glucose levels were $<7.77 \mathrm{mmol} / 1$ into quintiles and calculated multivariate adjusted HRs of CVD mortality to check for a threshold within normal and higher normal CBG. We used a Cox proportional hazard model adjusted for age, total cholesterol, BMI, hypertension, smoking categories, drinking categories and residential districts.

Population-attributable fractions (PAFs) for CHD, all heart disease, CVD and all-cause mortality were calculated as $\mathrm{pd} \times(\mathrm{HR}-1) / \mathrm{HR}$ [22], where $\mathrm{pd}$ is the proportion of death cases in the groups exposed to the risk, i.e. the borderline high CBG groups and the high CBG groups, and the multiple-adjusted HRs were used for the calculations. When calculating the PAF of the borderline high CBG group alone, the results for the high CBG group were not included. We calculated all PAFs using the combined dataset of men and women.

All confidence intervals were estimated at the $95 \%$ level. A $p$ value of $<0.05$ was considered significant. The Statistical Package for the Social Science (version 11.0J; SPSS Japan, Tokyo, Japan) was used for the analysis.

\section{Results}

The age (mean $\pm \mathrm{SD})$ at the baseline survey for all participants was $50.4 \pm 13.2$ for men and $50.8 \pm 13.3$ for women. The mean level of adjusted serum glucose with the hexokinase method was $5.67 \pm 1.82 \mathrm{mmol} / 1$ for men and $5.59 \pm 1.60 \mathrm{mmol} / \mathrm{l}$ for women. Table 1 shows baseline 
Table 1 Prevalence characteristics stratified by CBG levels at the baseline survey in 1980, NIPPON DATA80

\begin{tabular}{|c|c|c|c|c|c|}
\hline & \multicolumn{5}{|c|}{ CBG levels at baseline $(\mathrm{mmol} / \mathrm{l})$} \\
\hline & $\begin{array}{l}\text { Lower normal } \\
(<5.22)\end{array}$ & $\begin{array}{l}\text { Higher normal } \\
(5.22 \leq \mathrm{CBG}<7.77)\end{array}$ & $\begin{array}{l}\text { Borderline high } \\
(7.77 \leq \mathrm{CBG}<11.1)\end{array}$ & $\begin{array}{l}\operatorname{High}^{\mathrm{a}} \\
(\geq 11.1)\end{array}$ & $p$ value \\
\hline \multicolumn{6}{|l|}{ Men } \\
\hline$n$ & 1,915 & 1,814 & 187 & 218 & $<0.01$ \\
\hline Age (years) & $48.3 \pm 12.2$ & $51.3 \pm 13.7$ & $54.8 \pm 14.0$ & $57.0 \pm 11.1$ & $<0.01$ \\
\hline $\mathrm{S}$ glucose $(\mathrm{mmol} / \mathrm{l})$ & $4.64 \pm 0.50$ & $6.02 \pm 0.64$ & $8.83 \pm 0.90$ & $9.14 \pm 5.03$ & $<0.01$ \\
\hline $\mathrm{S}$ total chol $(\mathrm{mmol} / \mathrm{l})$ & $4.80 \pm 0.81$ & $4.80 \pm 0.86$ & $4.80 \pm 0.94$ & $5.06 \pm 0.92$ & $<0.01$ \\
\hline Current smoker (\%) & 64.1 & 61.8 & 65.3 & 66.0 & 0.66 \\
\hline Current drinker (\%) & 77.0 & 73.2 & 66.3 & 73.0 & $<0.01$ \\
\hline Hypertension (\%) & 43.1 & 54.3 & 61.5 & 67.0 & $<0.01$ \\
\hline Obesity $(\%)$ & 18.3 & 19.8 & 17.6 & 24.8 & 0.11 \\
\hline \multicolumn{6}{|l|}{ Residential districts } \\
\hline Urban $(\%)$ & 31.7 & 31.0 & 28.9 & 35.3 & 0.19 \\
\hline Suburban $(\%)$ & 24.8 & 22.9 & 21.9 & 23.9 & \\
\hline Sub-rural (\%) & 15.7 & 15.0 & 15.5 & 15.6 & \\
\hline Rural (\%) & 25.0 & 28.9 & 29.9 & 21.1 & \\
\hline \multicolumn{6}{|l|}{ Women } \\
\hline$n$ & 2526 & 2467 & 175 & 142 & $<0.01$ \\
\hline Age (years) & $47.4 \pm 12.5$ & $53.5 \pm 13.3$ & $56.7 \pm 11.6$ & $60.3 \pm 11.5$ & $<0.01$ \\
\hline $\mathrm{S}$ glucose $(\mathrm{mmol} / \mathrm{l})$ & $4.67 \pm 0.44$ & $6.00 \pm 0.60$ & $8.95 \pm 0.88$ & $10.7 \pm 5.22$ & $<0.01$ \\
\hline $\mathrm{S}$ total chol $(\mathrm{mmol} / \mathrm{l})$ & $4.83 \pm 0.84$ & $5.02 \pm 0.91$ & $5.12 \pm 0.86$ & $5.32 \pm 0.86$ & $<0.01$ \\
\hline Current smoker (\%) & 9.0 & 8.6 & 9.7 & 9.1 & 0.54 \\
\hline Current drinker (\%) & 21.4 & 19.0 & 16.0 & 15.5 & 0.19 \\
\hline Hypertension (\%) & 32.1 & 48.3 & 61.1 & 69.0 & $<0.01$ \\
\hline Obesity $(\%)$ & 19.6 & 25.2 & 25.7 & 36.6 & $<0.01$ \\
\hline \multicolumn{6}{|l|}{ Residential districts } \\
\hline Urban $(\%)$ & 31.7 & 32.3 & 31.4 & 32.4 & $<0.01$ \\
\hline Suburban (\%) & 26.4 & 21.4 & 26.3 & 26.8 & \\
\hline Sub-rural (\%) & 16.0 & 15.0 & 15.4 & 15.5 & \\
\hline Rural (\%) & 23.4 & 28.6 & 25.7 & 23.2 & \\
\hline
\end{tabular}

Unless indicated otherwise, values are means $( \pm \mathrm{SD})$

ANOVA was used for comparisons of multiple group means and the $\chi^{2}$ test was used to compare frequencies

Hypertension was defined as systolic blood pressure $\geq 140 \mathrm{mmHg}$, diastolic pressure $\geq 90 \mathrm{mmHg}$, the use of antihypertensive agents or any combination of these. Obesity was defined as BMI $\geq 25 \mathrm{~kg} / \mathrm{m}^{2}$

${ }^{a}$ Participants with a history of diabetes mellitus were placed in the high CBG group

$\mathrm{S}$ glucose, serum glucose; $\mathrm{S}$ total chol, serum total cholesterol

characteristics of the participants according to the serum glucose category. The higher glucose groups for both sexes had a higher age and higher prevalence of hypertension than lower glucose groups. Serum total cholesterol mean values for women were higher in the higher glucose groups. The prevalence of current smoking for men was higher in the higher glucose groups, but this difference was not statistically significant. The prevalence of obesity was highest in the high CBG groups in both sexes. The prevalence of current drinking and the residential districts for both sexes was not associated with glucose categories.

Diabetes prevalence in our data per 10 year age groups was $1.0 \%$ for those in their $30 \mathrm{~s}$ and $2.4,5.0,7.2,7.3$ and $4.8 \%$ for those in their $40 \mathrm{~s}, 50 \mathrm{~s}, 60 \mathrm{~s}, 70 \mathrm{~s}$ and $80 \mathrm{~s}$, respectively. When borderline high CBG was included, the prevalence was $3.1,5.4,9.7,13.0,12.8$ and $11.6 \%$ for the age groups above. Total person-years were 163,044 (69,946 for men, 93,098 for women) and the mean followup period was 17.3 years. During follow-up, we observed 1,911 all-cause deaths (1,025 for men, 886 for women), 137 CHD deaths (68 men, 69 women), 336 all heart diseases (164 men, 172 women) and 692 CVD deaths (345 men, 347 women).

Figure 1 shows age- and sex-adjusted HRs for CHD mortality of participants whose blood glucose levels for given time categories since last meal were $\geq 7.77 \mathrm{mmol} / 1$ compared with those whose CBG levels were $<7.77 \mathrm{mmol} / 1$. All HRs were positive regardless of the time since the last meal, though the HR was not statistically significant in the $1 \mathrm{~h}$ since last meal category. 


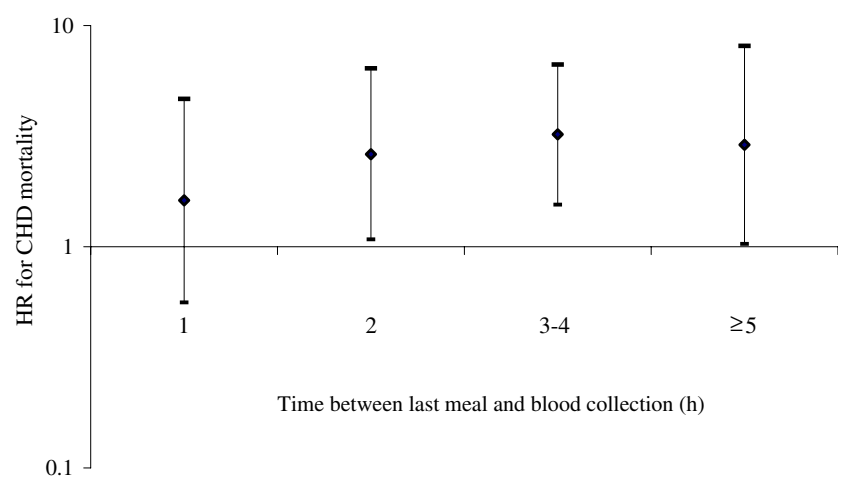

Fig. 1 Age- and sex-adjusted HRs with 95\% CIs for CHD deaths in participants whose CBG levels were $\geq 7.77 \mathrm{mmol} / 1$ or who selfreported diabetes mellitus compared with those whose CBG levels were $<7.77 \mathrm{mmol} / \mathrm{l}$. Results are grouped according to time in hours between last meal and blood collection time, and a log scale was used. Participants per time category: $n=1,164,1,148,2,822$ and 4,310 for 1 , $2,3-4$ and $\geq 5$ h, respectively. HRs per time category: $H R=1.62,2.62$, 3.22 and 2.89 for $1,2,3-4$ and $\geq 5$ h, respectively

Table 2 shows the number of deaths and multivariate adjusted HRs for CHD, all heart diseases, CVD and allcause mortality. The crude CHD mortality rate was 0.84 per 1,000 person-years in men and women combined ( 0.97 for men, 0.74 for women). HRs for any cause-specific CVD mortality suggested a graded relationship with glucose categories. HR values for CHD mortality were consistently higher in the high $\mathrm{CBG}$ and the borderline high $\mathrm{CBG}$ groups than in the reference groups. The HRs for CHD mortality were also higher in the higher normal blood glucose groups than in the reference groups. HRs for CVD and all heart diseases mortality showed similar tendencies. Because no interaction between sex and CBG was observed for CVD $(p=0.91)$, we combined men and women in the following analyses. When we combined lower normal and higher normal into one category as the reference group (i.e. $\mathrm{CBG} \leq 7.77 \mathrm{mmol} / \mathrm{l})$, the HRs for CHD mortality were 2.12 (95\% CI 1.19-3.79) for borderline high CBG and 2.29 (95\% CI 1.36-3.86) for high CBG. Similar findings were observed in both sexes (data not shown). Even when we restricted the analysis to participants with values within the normal blood glucose range $(\mathrm{CBG} \leq 7.77 \mathrm{mmol} / \mathrm{l})$, each $1 \mathrm{mmol} / 1$ increase in CBG was associated with a significant increase in the HR for CVD mortality (HR 1.12, 95\% CI 1.02-1.22). The HR for CHD mortality per $1 \mathrm{mmol} / \mathrm{l}$ increase in $\mathrm{CBG}$ was similar to that for CVD, but this increase was not significant (HR 1.09, 95\% CI 0.89-1.34). When we divided participants within the normal blood glucose range $(\mathrm{CBG} \leq 7.77 \mathrm{mmol} / \mathrm{l})$ into quintiles, HRs for CVD mortality

Table 2 The number of deaths and multivariate-adjusted HRs for CHD, all heart diseases, CVD and all-cause mortality according to CBG levels

\begin{tabular}{|c|c|c|c|c|}
\hline & \multicolumn{4}{|c|}{ Baseline serum CBG, men and women combined } \\
\hline & Lower normal & Higher normal & Borderline high & High \\
\hline Glucose (mmol/l) & $<5.22$ & $5.22 \leq \mathrm{CBG}<7.77$ & $7.77 \leq \mathrm{CBG}<11.1$ & $\geq 11.10$ \\
\hline$n$ & 4,441 & 4,281 & 362 & 360 \\
\hline Person-years & 78,635 & 73,005 & 5,939 & 5,465 \\
\hline \multicolumn{5}{|l|}{ Cause of death } \\
\hline \multicolumn{5}{|l|}{ CHD } \\
\hline Deaths $(n)$ & 38 & 69 & 13 & 17 \\
\hline Mortality rate ${ }^{\mathrm{a}}$ & 0.5 & 0.9 & 2.2 & 3.1 \\
\hline $\operatorname{HR}(95 \% \mathrm{CI})^{\mathrm{b}}$ & 1 & $1.24(0.83-1.86)$ & $2.43(1.29-4.58)$ & $2.62(1.46-4.67)$ \\
\hline \multicolumn{5}{|l|}{ All heart diseases } \\
\hline Deaths $(n)$ & 105 & 167 & 29 & 35 \\
\hline Mortality rate $^{\mathrm{a}}$ & 1.3 & 2.3 & 4.9 & 6.4 \\
\hline $\operatorname{HR}(95 \% \mathrm{CI})^{\mathrm{b}}$ & 1 & $1.06(0.83-1.36)$ & $1.78(1.17-2.70)$ & $2.07(1.41-3.06)$ \\
\hline \multicolumn{5}{|l|}{ CVD } \\
\hline Deaths $(n)$ & 205 & 379 & 47 & 61 \\
\hline Mortality rate $^{\mathrm{a}}$ & 2.6 & 5.2 & 7.9 & 11.2 \\
\hline $\operatorname{HR}(95 \% \mathrm{CI})^{\mathrm{b}}$ & 1 & $1.22(1.03-1.45)$ & $1.46(1.06-2.01)$ & $1.82(1.37-2.43)$ \\
\hline \multicolumn{5}{|l|}{ All causes } \\
\hline Deaths $(n)$ & 665 & 976 & 107 & 163 \\
\hline Mortality rate $^{\mathrm{a}}$ & 8.5 & 13.4 & 18.0 & 29.8 \\
\hline $\operatorname{HR}(95 \% \mathrm{CI})^{\mathrm{b}}$ & 1 & $1.07(0.96-1.18)$ & $1.13(0.92-1.38)$ & $1.63(1.37-1.93)$ \\
\hline
\end{tabular}

Sex was included in the model, as the combined dataset of men and women was analysed

Hypertension was defined as systolic blood pressure $\geq 140 \mathrm{mmHg}$, diastolic blood pressure $\geq 90 \mathrm{mmHg}$, the use of antihypertensive agents or any combination of these

${ }^{\mathrm{a}}$ The mortality rate is the crude mortality rate per 1,000 person-years

${ }^{\mathrm{b}}$ The HRs were adjusted for age, serum total cholesterol levels, BMI, hypertension, cigarette smoking categories, drinking categories and residential districts. $p<0.001$ for all trends 
increased gradually in the high CBG groups $(p<0.01$ for trend; Table 3). PAFs of high CBG and borderline high CBG for CHD, all heart diseases, CVD and all-cause mortality were $12.0,8.8,4.9$ and $3.5 \%$, respectively, compared with normal $\mathrm{CBG}$ groups $(\mathrm{CBG}<7.77 \mathrm{mmol} / \mathrm{l})$. PAFs of high $\mathrm{CBG}$ alone for $\mathrm{CHD}$, all heart diseases, CVD and all-cause mortality were $7.0,5.2,3.3$ and $3.1 \%$, respectively. PAFs of the borderline high $\mathrm{CBG}$ alone for $\mathrm{CHD}$, all heart diseases, CVD and all-cause mortality were $5.0,3.6,1.5$ and $0.4 \%$, respectively.

\section{Discussion}

In this study, we found that CBG levels predicted CHD mortality. The risk for CHD mortality increases with $\mathrm{CBG}$ levels, regardless of time since last meal. We also found that participants with borderline high $\mathrm{CBG}$ or high $\mathrm{CBG}$ had higher mortality due to CHD. Furthermore, the data showed that lower blood glucose levels led to lower HRs for CVD mortality, even within the normal blood glucose range.

Our cohort study was conducted with a representative sample from the Japanese population with a long-term follow-up period. The participants were randomly selected from across Japan. The participation rate and follow-up rate were high: more than 75 and $90 \%$, respectively. Thus our data should be applicable to the general Japanese popula-

Table 3 The number of deaths and multivariate-adjusted HRs for CVD according to CBG levels $<7.77 \mathrm{mmol} / 1$ divided into five classes, for men and women combined

Cardiovascular diseases

\begin{tabular}{|c|c|c|c|c|c|}
\hline $\begin{array}{l}\text { Baseline } \\
\text { CBG } \\
(\mathrm{mmol} / \mathrm{l})\end{array}$ & $n$ & $\begin{array}{l}\text { Person- } \\
\text { years }\end{array}$ & $\begin{array}{l}\text { Deaths } \\
(n)\end{array}$ & $\begin{array}{l}\text { Mortality } \\
\text { rate }^{\mathrm{a}}\end{array}$ & $\begin{array}{l}\text { HR } \\
(95 \% \mathrm{CI})^{\mathrm{b}}\end{array}$ \\
\hline $1.40-4.63$ & 1,710 & 31,552 & 71 & 2.3 & 1 \\
\hline $4.68-5.01$ & 1,766 & 32,905 & 84 & 2.6 & $\begin{array}{l}1.07 \\
(0.78-1.47)\end{array}$ \\
\hline $5.06-5.44$ & 1,632 & 30,485 & 117 & 3.8 & $\begin{array}{l}1.30 \\
(0.97-1.75)\end{array}$ \\
\hline $5.48-6.01$ & 1,579 & 29,826 & 141 & 4.7 & $\begin{array}{l}1.33 \\
(1.00-1.78)\end{array}$ \\
\hline $6.05-7.76$ & 1,603 & 29,655 & 193 & 6.5 & $\begin{array}{l}1.39 \\
(1.06-1.83)\end{array}$ \\
\hline
\end{tabular}

Hypertension was defined as systolic blood pressure $\geq 140 \mathrm{mmHg}$, diastolic blood pressure $\geq 90 \mathrm{mmHg}$, the use of antihypertensive agents or any combination of these

The HRs were adjusted for age, sex, serum total cholesterol levels, BMI, hypertension, cigarette smoking categories, drinking categories and residential districts

${ }^{a}$ The mortality rate is the crude mortality rate per 1,000 person-years

${ }^{\mathrm{b}} p$ for trend $<0.01$ tion. Due to our study's representative nature, we were able to assess the PAF of high CBG and borderline high CBG groups for CHD mortality.

The prevalence of diabetes mellitus in our data at baseline was comparable with that reported in other studies. Sairenchi et al. [23] reported that the diabetes prevalence in men aged $40-59$ at baseline was $6.2 \%$ in those who survived, $8.4 \%$ in participants who died of CVD and $12.4 \%$ in those who died of non-CVD causes during a follow-up period of 9 years. The prevalence for women was $2.6,11.4$ and $5.4 \%$, respectively. The corresponding prevalence in those aged 60-79 at baseline was 7.8, 11.4 and $10.7 \%$ for men and 5.2, 12.2 and $7.4 \%$ for women. The investigators collected nonfasting samples and defined diabetes mellitus as a plasma glucose level $\geq 7.0 \mathrm{mmol} / \mathrm{l}$ (fasting), $\geq 11.1 \mathrm{mmol} / 1$ (nonfasting) or treatment for diabetes mellitus. We believe that these results are consistent with ours. As expected, CHD mortality rates from our data were very low compared with those of other developed countries. Other papers have also pointed out the low CHD mortality in Japan [24, 25]. Although the reason is unclear, various hypotheses have been proposed, e.g. low serum cholesterol levels in Japanese in 1980 compared with westernised countries [11], as well as levels of fish [26] or green tea intake [27] among the Japanese.

In the present study, we were able to show that $\mathrm{CBG}$ potentially predicts future CVD mortality. Thus, in some settings, such as community screenings for CVD risk factors, where high participation rates are desired, as well as in clinics normally not visited in a fasting state, CBG could be a viable alternative to OGTT or fasting blood glucose. Our finding on prediction of CVD mortality was also consistent with previous reports. Based on the NIPPON DATA80, the probability of death over a 10 year period from CHD, stroke and CVD was calculated and displayed as colour charts [10]. These charts showed that participants with diabetes mellitus defined as CBG levels $\geq 11.1 \mathrm{mmol} / \mathrm{l}$ had higher CHD, stroke and CVD mortality risks than those without diabetes mellitus. Our results expanded on those results. Irie et al. [6] reported the relationship between $\mathrm{CBG}$ obtained in general health check-ups and CHD, CVD and all-cause mortality in a Japanese population. Their study had a large sample with a shorter follow-up period of 5 years. Similarly to our results, they found that the HRs for CHD, CVD and all-cause mortality were higher than the reference groups, even among participants in the borderline high CBG group.

It should, however, be emphasised that although CBG is a feasible way of assessing CVD risk factors in community screenings, it cannot be used for a definitive diagnosis of diabetes because of the difficulty in standardisation. Recently, diabetes risk scores incorporating age, sex, BMI, steroid or antihypertensive medication, family history, 
smoking history and other factors have been accepted as the most appropriate tool for initial diabetes mellitus screening [28-30]. The use of such a risk score might also be feasible for initial screening, although its value has yet to be established in the Japanese population.

In our study, HRs for CVD mortality were higher in participants in the higher normal blood glucose group than in those in the lower normal group. Furthermore, a statistically significant positive linear relationship was observed within normal glucose levels. The HRs for CVD mortality seemed to increase linearly when we divided normal CBG $(<7.77 \mathrm{mmol} / \mathrm{l})$ into quintiles. We considered the relationship between CBG and CVD risk as continuous, rather than being determined by a threshold. Thus, similarly to blood pressure [31-33], lower CBG may yield lower CVD mortality rates, as some epidemiological studies have shown in meta-analyses with fasting blood glucose [34] or in other studies with OGTT $[9,35]$.

From a public health perspective, these results suggest that there is a certain impact on excess risk of CVD death, even when serum blood glucose increases mildly, without reaching the borderline high CBG level. In our study, the number of participants with higher normal glucose was much greater than that for those with borderline high CBG and high CBG. As a population strategy, lifestyle modifications such as body weight reduction, smoking cessation and an increase in physical activity may be effective, less intensive ways to improve a higher normal glucose level [36]. Other strategies such as adequate medication and/or intensive lifestyle modifications might reduce the risk of CVD mortality for individuals with borderline high CBG or high CBG.

The present study has a number of limitations. First, since it was based on blood glucose level measurement on one occasion only, the results might include a regression dilution bias, possibly attenuating the association between CBG and long-term mortality [37]. Second, since we used death as an endpoint, we only estimated fatal CVD and did not include non-fatal cases. Third, since we had no information on fasting blood glucose levels or post load glucose obtained by OGTT results, we were unable to compare the predictive power of the different methods for assessing blood glucose levels. Finally, socioeconomic status might have affected our results, but we were unable to adjust for this factor owing to a lack of relevant information. In conclusion, CBG predicted CHD and CVD mortality in a Japanese population regardless of time since last meal. Even within the normal range, raised CBG levels were related to an elevated risk of CHD and CVD mortality in Japanese. Thus, CBG could be an alternative to fasting blood glucose or OGTT, in situations where it is unrealistic to ask all patients to fast, as in population screening for CVD risk factors, which requires higher participation rates.
Acknowledgements This study was supported by a grant-in-aid of the Japanese Ministry of Health and Welfare under the auspices of the Japanese Association for Cerebro-cardiovascular Disease Control, a Research Grant for Cardiovascular disease (7A-2) from the Ministry of Health, Labor and Welfare and a Health and Labor Sciences Research Grant, Japan (Comprehensive Research on Aging and Health: H11-Chouju-046, H14-Chouju-003, H17-Chouju-012).

Duality of interest The authors declare that there is no duality of interest associated with this manuscript.

\section{References}

1. Fuller JH, Shipley MJ, Rose G, Jarrett RJ, Keen H (1983) Mortality from coronary heart disease and stroke in relation to degree of glycaemia: the Whitehall study. BMJ 287:867-870

2. Kannel WB, Mcgee DL (1979) Diabetes and cardiovascular disease: the Framingham study. JAMA 241:2035-2038

3. Stamler J, Vaccaro O, Neaton JD, Wentworth D (1993) Diabetes, other risk factors, and 12-yr cardiovascular mortality for men screened in the Multiple Risk Factor Intervention Trial. Diabetes Care 16:434-444

4. Levitan EB, Song Y, Ford ES, Liu S (2004) Is nondiabetic hyperglycemia a risk factor for cardiovascular disease? A metaanalysis of prospective studies. Arch Intern Med 164:2147-2155

5. Expert Committee on the Diagnosis and Classification of Diabetes Mellitus (2003) Report of the expert committee on the diagnosis and classification of diabetes mellitus. Diabetes Care 26(Suppl 1): S5-S20

6. Irie F, Sairenchi T, Iso H, Shimamoto T (2001) Prediction of mortality from findings of annual health checkups utility for health care programs. Nippon Koshu Eisei Zasshi 48:95-108 (article in Japanese)

7. Fujishima M, Kiyohara Y, Kato I et al (1996) Diabetes and cardiovascular disease in a prospective population survey in Japan: The Hisayama Study. Diabetes 45(Suppl 3):S14-S16

8. Meigs JB, Nathan DM, D'Agostino RB Sr, Wilson PW (2002) Framingham offspring study: fasting and postchallenge glycemia and cardiovascular disease risk: the Framingham Offspring study. Diabetes Care 25:1845-1850

9. Brunner EJ, Shipley MJ, Witte DR, Fuller JH, Marmot MG (2006) Relation between blood glucose and coronary mortality over 33 years in the Whitehall Study. Diabetes Care 29:26-31

10. NIPPON DATA80 Research Group (2006) Risk assessment chart for death from cardiovascular disease based on a 19-year followup study of a Japanese representative population. Circ J 70:12491255

11. Okamura $\mathrm{T}$, Tanaka H, Miyamatsu N, for NIPPON DATA80 Research Group (2007) The relationship between serum total cholesterol and all-cause or cause-specific mortality in a 17.3-year study of a Japanese cohort. Atherosclerosis 190:216-223

12. Ueshima H, Choudhury SR, Okayama A et al (2004) Cigarette smoking as a risk factor for stroke death in Japan: NIPPON DATA80. Stroke 35:1836-1841

13. Okamura T, Hayakawa T, Kadowaki T, et al., for NIPPON DATA80 research group (2004) A combination of serum low albumin and above-average cholesterol level was associated with excess mortality. J Clin Epidemiol 57:1188-1195

14. Okamura T, Kadowaki T, Hayakawa T, Kita Y, Okayama A, Ueshima H, for Nippon Data80 Research Group (2003) What cause of mortality can we predict by cholesterol screening in the Japanese general population? J Intern Med 253:169-180 
15. Nakamura M, Sato S, Shimamoto T (2003) Improvement in Japanese clinical laboratory measurements of total cholesterol and HDL-cholesterol by the US Cholesterol Reference Method Laboratory Network. J Atheroscler Thromb 10:145-153

16. Bittner D, McCleary M (1963) The cupric-phenanthroline chelate in the determination of monosaccharides in whole blood. Am J Clin Pathol 40:423-424

17. Iso H, Imano H, Kitamura A et al (2004) Type 2 diabetes and risk of non-embolic ischaemic stroke in Japanese men and women. Diabetologia 47:2137-2144

18. Shirai K (2001) Evaluation of obesity and diagnostic criteria of obesity as a disease for Japanese (in Japanese). Nippon Rinsho 59:578-585

19. Nishi N, Sugiyama H, Kasagi F et al (2007) Urban-rural difference in stroke mortality from a 19-year cohort study of the Japanese general population: NIPPON DATA80. Soc Sci Med 65:822-832

20. Okamura T, Hayakawa T, Kadowaki T, for NIPPON DATA80 Research Group (2004) Resting heart rate and cause-specific death in a 16.5-year cohort study of the Japanese general population. Am Heart J 147:1024-1032

21. American Diabetes Association (2005) Diagnosis and classification of diabetes mellitus. Diabetes Care 28(Suppl 1):S37-S42

22. Rockhill B, Newman B, Weinberg C (1998) Use and misuse of population attributable fractions. Am J Public Health 88:15-19

23. Sairenchi T, Iso H, Irie F et al (2005) Age-specific relationship between blood pressure and the risk of total and cardiovascular mortality in Japanese men and women. Hypertens Res 28:901-909

24. Sekikawa A, Satoh T, Hayakawa T, Ueshima H, Kuller LH (2001) Coronary heart disease mortality among men aged 35-44 years by prefecture in Japan in 1995-1999 compared with that among white men aged 35-44 by state in the United States in 1995-1998: vital statistics data in recent birth cohort. Jpn Circ J 65:887-892

25. Sekikawa A, Horiuchi BY, Edmundowicz D et al (2003) A "natural experiment" in cardiovascular epidemiology in the early 21st century. Heart 89:255-257

26. Sekikawa A, Ueshima H, Zaky WR et al (2005) Much lower prevalence of coronary calcium detected by electron-beam computed tomography among men aged 40-49 in Japan than in the US, despite a less favorable profile of major risk factors. Int J Epidemiol 34:173-179

27. Kuriyama S, Shimazu T, Ohmori K et al (2006) Green tea consumption and mortality due to cardiovascular disease, cancer, and all causes in Japan: the Ohsaki Study. JAMA 296:1255-1265

28. Griffin SJ, Little PS, Hales CN, Kinmonth AL, Wareham NJ (2000) Diabetes risk score: towards earlier detection of type 2 diabetes in general practice. Diabetes Metab Res Rev 16:164-171

29. Lindstrom J, Tuomilehto J (2003) The diabetes risk score: a practical tool to predict type 2 diabetes risk. Diabetes Care 26:725-731

30. Franciosi M, De Berardis G, Rossi MC et al (2005) Use of the diabetes risk score for opportunistic screening of undiagnosed diabetes and impaired glucose tolerance: the IGLOO (Impaired Glucose Tolerance and Long-Term Outcomes Observational) Study. Diabetes Care 28:1187-1194

31. Lawes CM, Bennett DA, Feigin VL, Rodgers A (2004) Blood pressure and stroke: an overview of published reviews. Stroke 35:776-785

32. Asia Pacific Cohort Studies Collaboration (2003) Blood pressure and cardiovascular disease in the Asia Pacific region. J Hypertens 21:707-716

33. Prospective Studies Collaboration (2002) Age-specific relevance of usual blood pressure to vascular mortality: a meta-analysis of individual data for one million adults in 61 prospective studies. Lancet 360:1903-1913

34. Lawes CM, Parag V, Bennett DA, for Asia Pacific Cohort Studies Collaboration (2004) Blood glucose and risk of cardiovascular disease in the Asia Pacific region. Diabetes Care 27:2836-2842

35. Rodriguez BL, Lau N, Burchfiel CM et al (1999) Glucose intolerance and 23-year risk of coronary heart disease and total mortality: the Honolulu Heart Program. Diabetes Care 22:1262 1265

36. Rose G (1992) The strategy of preventive medicine. Oxford University Press, Oxford

37. MacMahon S, Peto R, Cutler J et al (1990) Blood pressure, stroke, and coronary heart disease. Part 1, Prolonged differences in blood pressure: prospective observational studies corrected for the regression dilution bias. Lancet 335:765-774 